\title{
Age-related differences in selection by visual saliency
}

\author{
Kamen A. Tsvetanov • Carmel Mevorach • \\ Harriet Allen • Glyn W. Humphreys
}

Published online: 29 June 2013

(C) Psychonomic Society, Inc. 2013

\begin{abstract}
We examined the ability of older adults to select local and global stimuli varying in perceptual saliency-a task requiring nonspatial visual selection. Participants were asked to identify in separate blocks a target at either the global or the local level of a hierarchical stimulus, while the saliency of each level was varied (across different conditions, either the local or the global form was the more salient and relatively easier to identify). Older adults were less efficient than young adults in ignoring distractors that were higher in saliency than were targets, and this occurred across both the global and local levels of form. The increased effects of distractor saliency on older adults occurred even when the effects were scaled by overall differences in task performance. The data provide evidence for an age-related decline in nonspatial attentional selection of low-salient hierarchical stimuli, not determined by the (global or local) level at which selection was required. We discuss the implications of these results for understanding both the interaction between saliency and hierarchical processing and the effects of aging on nonspatial visual attention.
\end{abstract}

Keywords Saliency · Nonspatial visual attention · Aging · Global and local processing · Inhibition deficit theory · Distraction

K. A. Tsvetanov $(\bowtie) \cdot$ C. Mevorach

School of Psychology, University of Birmingham, Birmingham B15 2TT, UK

e-mail: k.tsvetanov@bham.ac.uk

H. Allen

School of Psychology, University of Nottingham, Nottingham NG7

2RD, UK

G. W. Humphreys

Department of Experimental Psychology, University of Oxford,

Oxford OX1 3UD, UK

\section{Introduction}

In order to survive in complex, dynamic environments, we need efficient mechanisms of attention to select information relevant to our behavioral goals. Current theories of visual attention hold that selection is determined by the interaction between bottom-up and top-down signals. Bottom-up signals act to draw attention to salient items that differ from their local surroundings (Theeuwes, 1992, 2005). Top-down forms of selection become involved when participants have particular expectations about the target they are required to select (e.g., knowing its location or one of its features; Wolfe, Butcher, Lee, \& Hyle, 2003) and/or when the target is less salient than particular distractors - when the bottom-up attraction of attention to the salient distractors must be overcome. In addition, top-down selection itself can be fractionated into excitatory processes, which guide attention to targets, and inhibitory processes, which can filter out irrelevant distractors (see Braithwaite et al., 2005; Dent et al., 2012). There is evidence that the mechanisms of selection operate less efficiently as we age (Madden, Gottlob, \& Allen, 1999; Plude, 1990), but the interplay of bottom-up and top-down processes in the effects of aging remain poorly understood (see Madden, 2007). For example, there is evidence that the role of top-down expectations for targets and of excitatory guidance may be stronger in older than in younger adults (Madden et al., 1999). On the other hand, the ability to suppress irrelevant distractors may decrease, as argued by the inhibition deficit theory of cognitive aging (Lustig, Hasher, \& Zacks, 2007). According to this account, cognitive aging is associated with a selective decrease in the ability to inhibit irrelevant stimuli and responses, worsening attentional selection in older adults. Although the loss of inhibitory control may be compensated for by increased top-down excitatory guidance, problems will emerge under conditions in which distractors strongly compete for selection with targets (e.g., under conditions in which distractors have the higher saliency). 
In the present study, we set out to examine the interplay between bottom-up and top-down processing by examining the effects of aging on the ability to select stimuli on the basis of their relative saliency. The relative saliency of a stimulus will reflect the strength of its bottom-up representation, as compared with other stimuli in the field. The bottom-up saliency of a stimulus can then either match or be pitched against top-down drivers of selection, according to whether the target or a distractor stimulus has the higher relative salience. The effects of responding to bottom-up saliency can be assessed by examining the selection of high-saliency targets accompanied by low-saliency distractors. In contrast, the strength of top-down control of selection can be assessed by performance when the target has low saliency and the distractor high saliency. Here, we ask whether aging differentially affects either the guidance of attention to salient targets (e.g., due to reduced sensitivity to bottom-up salience) or the rejection of salient distractors when lowsaliency targets are selected (due to inhibitory deficits affecting top-down modulation of selection).

\section{Inhibitory deficits in visual selection}

Evidence for the inhibitory deficit theory emerges from studies using negative priming. Negative-priming tasks measure the unfavorable influence of a prior exposure to a distractor stimulus on the response to the same stimulus when a target. Classic studies of negative priming have contrasted cases where an item's identity is inhibited, slowing its subsequent identification (Tipper \& Cranston, 1985). Reduced negative priming in older adults might reflect less efficient inhibition when the stimuli are first encountered as distractors (Hasher, Stoltzfus, Zacks, \& Rypma, 1991). In addition, there is evidence for age-related changes in location-based inhibition - for example, in preview search tasks. Preview search typically uses conjunction-like displays but presents distractors with one common set of properties prior to the second set of distractors plus the target (Watson \& Humphreys, 1997). Provided there is a sufficient period between the two sets of distractors (of the order of $400 \mathrm{~ms}$ or so), the first set of distractors can be efficiently ignored (Humphreys, Stalmann, \& Olivers, 2004). There is substantial evidence that the lack of impact of the initial distractors is dependent, at least in part, on a process of active distractor suppression of its location (Allen, Humphreys, \& Matthews, 2008; Humphreys et al., 2004; Watson \& Humphreys, 2000) and features (Olivers \& Humphreys, 2003). As in studies of negative priming, it has been shown that older participants recruit distinctly different functional mechanisms (Allen \& Payne, 2011) and can show a selective reduction in the efficiency of preview search, particularly under conditions where distractor inhibition is challenged (e.g., with moving distractors; Watson \& Maylor, 2002).
This is consistent with reduced inhibition of distractor locations and/or features in older adults, relative to young adults.

However, there are many instances in everyday life where selection is neither feature nor space based but, rather, dependent on the ability to select the appropriate level of a form. For example, when trying to make a judgment about someone's identity, we may want to select the whole face in order to take advantage of configural relations between features, but in doing this, we may not want to attend to the local features themselves. On the other hand, when we make a judgment about part of a face (is the person smiling?), we may want to focus attention on the local part without processing the whole. For such cases, we need to be able to flexibly select the local or the global level of a form, an ability that likely depends on underlying mechanisms different from those studied through feature- or space-based selection. For example, while there is much evidence for spatial selection being dependent on a largely bilateral frontoparietal network (Corbetta \& Shulman, 2002), the selection of local and global forms has been associated with lateralized brain recruitment, with the left hemisphere being selectively linked to local processing and the right hemisphere to global processing (Lux et al., 2004). In addition, other regions may be recruited irrespective of whether the local or the global level of form needs to be selected, as a function of whether the target level (local or global) is high or low in salience. For example, the right posterior parietal cortex (PPC) has been linked to the guidance of attention toward the more salient of the levels when the target is at that level (Hodsoll, Mevorach, \& Humphreys, 2009), while the left PCC is involved when the target is at the less salient level and the distractor at the higher level of salience (Mevorach, Shalev, Allen, \& Humphreys, 2009). This PPC system has been linked also to a "top-down regulation" of the early visual regions, which would otherwise respond differentially to the salient stimulus (in this case, the distractor level). For example, Mevorach, Shalev, et al. used psycho-physiological interaction analysis to demonstrate that there was increased activation of the left PPC when high-saliency distractors had to be ignored, and this co-occurred with reduced activation of the left occipital cortex. They argued that the selection of the low-salient target was mediated by the left PPC suppressing distractorrelated activity in the early occipital cortex. In support of this, suppressive TMS to the left PPC led to increased activity in the occipital cortex when highly salient distractors were present, suggesting that there was then reduced topdown regulation of the occipital cortex. The behavioral evidence indicated that there was increased interference from salient distractors, and this effect was greatest when TMS was applied prior to the onset of the stimulus (Mevorach, Hodsoll, Allen, Shalev, \& Humphreys, 2010; Mevorach, Humphreys, \& Shalev, 2009). In this case, TMS appeared to block the top-down setting up of perceptual suppression. 
To date, work addressing the decreased ability to inhibit salient distractors, as a function of age, has been conducted under conditions of spatial selection, and we know little about how aging affects the ability to suppress irrelevant distractors when other forms of selection are demanded, such as selecting the level of form and/or selecting hierarchical stimuli according to their relative salience. Here, we set out to address this issue by evaluating how cognitive aging alters an individual's ability to select a low-saliency target in a hierarchical form, as compared with when the target is highly salient.

Global and local processing in aging

There are several previous studies of the effects of cognitive aging on the ability to select local and global levels of form. However, the results are very mixed. Roux and Ceccaldi (2001), for example, used stimuli that showed an overall global processing advantage and reported that older participants had stronger global interference (when responding to local targets) than did younger observers. In direct contrast, Muller-Oehring, Schulte, Raassi, Pfefferbaum, and Sullivan (2007), employing stimuli with an overall local advantage, found greater local-on-global interference in older participants. Others have reported null effects of aging on local and global interference (Bruyer, Scailquin, \& Samson, 2003). These contradictory results may be understood if cognitive aging affects the ability to select stimuli varying in saliency, rather than the ability to select local and global forms per se. For example, in studies showing greater interference effects in older participants, the interfering distractors were typically more salient than the target (global distractors in Roux \& Ceccaldi, 2001; local distractors in Muller-Oehring et al., 2007), while experiments showing no differential interference effects have tended to have local and global forms more balanced for saliency (e.g., as judged by overall RTs; Bruyer et al., 2003). The conflicting results may be accounted for by differential selection of stimuli varying in saliency, with older participants finding it particularly difficult to suppress highsalient distractors in order to select low-salient hierarchical targets (cf. Mevorach et al., 2010). We investigated this for the first time in this article, using stimuli modeled on investigations of selection by saliency by Mevorach and colleagues.

\section{Saliency processing in global/local level}

In contrast to prior studies in this field, Mevorach orthogonally varied whether the target was at the local or global level of the forms and whether it had high or low saliency (in relation to the distractor level of form) (Mevorach, Humphreys, \& Shalev, 2005, 2006a, 2006b, 2009; Mevorach, Shalev, et al., 2009). The saliency of the forms was varied either by presenting highcontrast local forms in alternating colors (high local saliency, low global saliency) or by blurring the hierarchical letter and presenting the local forms in uniform color (high global saliency, low local saliency; see Fig. 1). Performance was analyzed by pooling the data across conditions where the target was at the local level and when it was at the global level and contrasting the results when the target had high salience (and the distractor low salience) and when it had low salience (and the distractor high salience). When the target level was high in salience, the top-down demands on inhibition of the distractor level were low, since selection could have been driven by bottom-up cues; however, when the target was low in salience and the distractors had high salience, the top-down demands on distractor inhibition would be high in order to overcome bottom-up cues biasing selection in favor of the distractor.

\section{The present study}

In the present study, we report a novel analysis of selection by level of hierarchical form (global/local) and saliency in young and older adults, assessing whether older adults have

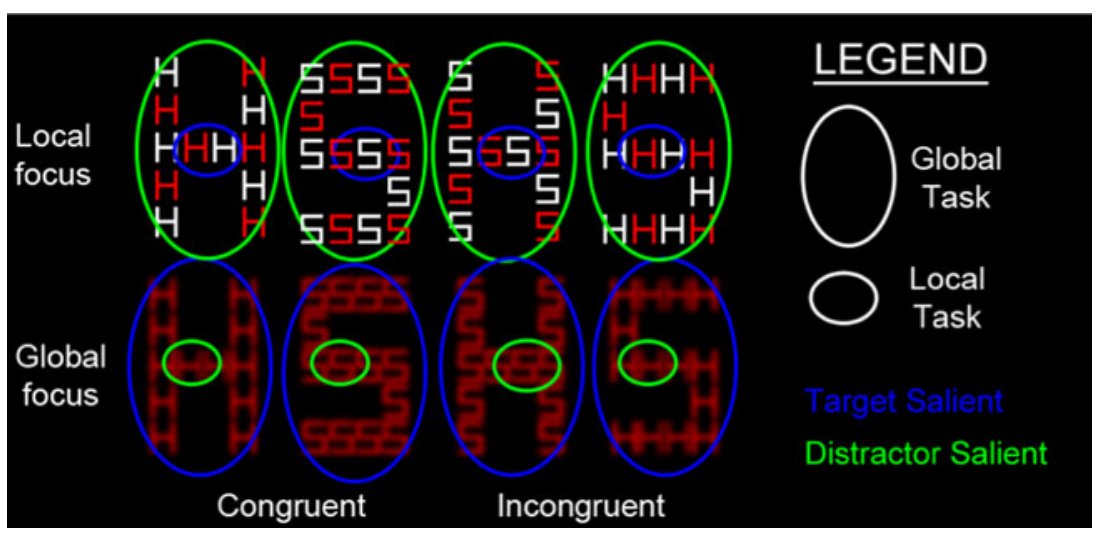

Fig. 1 All compound letters with either high local saliency (top row) or high global saliency (bottom row) used in the experiment. Target saliency was varied orthogonally with the task (local task [small circles]/global task [large ellipses]). The color of the ellipse denotes the saliency level in particular condition: target salient in blue (e.g., in a block with global task using the set of stimuli with global focus; bottom row) and distractor salient in green (e.g., in a block of local task using the set of stimuli with global focus; bottom row) 
particular problems selecting low-salient targets in the face of high-salient distractors and whether this occurs independently of the global or local level of the stimulus. To do this, we had participants select local and global letters under conditions of varying saliency using a blocked design, with either the target level being highly salient and the distractor having low saliency or the distractor level having high saliency and the target low (see Fig. 1). When the target has relatively high saliency, there should be bottom-up guidance of attention to targets coupled to a role of top-down excitatory guidance (to the blocked target level). On the other hand, when the target has relatively low saliency (and the distractor high saliency), there will be demands on top-down inhibition of the distractor level to prevent attention being drawn in a bottom-up manner to the distractor. We hypothesized that the ability to inhibit high-salient distractors for successful processing of low-salient targets is reduced with aging, in which case performance should be disrupted for older, relative to younger, adults when distractors have high saliency. On the other hand, if there is increased bottom-up attentional guidance and/or use of top-down excitatory guidance to targets, older participants should be relatively faster at selecting high-salient targets. By varying saliency orthogonally across the global and local levels of form, we tested too whether older adults had difficulties confined to one level of form or whether there was an overall effect of saliency that cut across the local and global stimuli.

\section{Method}

\section{Participants}

The participants were 24 young (11 males; group mean age, 24 years; age range, 19-29) and 19 older (10 males; group mean age, 74 years; age range, 65-84) healthy volunteers who received either course credits or cash $(£ 6 / h)$. The participants were recruited by advertisements in local communities, wordof-mouth information, and advertisements on an online experiment management system (Research Participation Scheme, University of Birmingham). All the participants had normal or corrected-to-normal vision (assessed in laboratory environment using Snellen chart) and were healthy with no history of psychiatric or neurological disease (self-report).

\section{Stimuli}

Two sets of compound-letter stimuli were created to have either high global saliency or high local saliency. The stimuli comprised the letters " $\mathrm{H}$ " and " $\mathrm{S}$," and their combinations created figures in the shape of large orthogonal "H" and "S" letters (see Fig. 1).

In the set of stimuli where local information was to be salient, the compound letters were made of red (RGB, 255
00 ) and white local letters (Fig. 1, top row). The size of the local letters was $1.34^{\circ} \times 1.76^{\circ}$ of visual angle (in width and height, respectively), with a distance between the letters of $0.46^{\circ}$. The total width and height of the global letters was $6.7^{\circ} \times 10.81^{\circ}$ of visual angle, respectively.

When the compound letters were weighted for global processing, the local stimuli consisted of red blurred letters (Fig. 1, bottom row). The width and the height of the local letters was $1.34^{\circ} \times 1.76^{\circ}$ of visual angle, respectively, with an interletter distance of $0.15^{\circ}$, resulting in a global letter subtending $5.83^{\circ} \times 9.22^{\circ}$ of visual angle (in width and height, respectively). These images were additionally blurred in MATLAB using a Gaussian low-pass filter (FWHM of $1.56 \mathrm{~mm}$ ). Mean display luminance for white, red, and black colors was $118.44,25.77$, and $>0.01$, respectively. Luminance measures were performed using a Minolta LS 110 photometer.

To reduce strategic focusing on a local area of the screen, there were three possible positions for presentation of the stimuli: the center or $13.16^{\circ}$ to the left or right of the center of the screen.

\section{Procedure}

In a selective attention task, participants undertook different trial blocks in which they were asked to concentrate only on the global or the local letters across a block of trials while ignoring the information at the other level. The task was to identify the letter ( $\mathrm{H}$ or $\mathrm{S}$ ) on the designated target level by pressing prespecified buttons on a USB mouse (e.g., "Is the letter on the global level H or S?"). The experiment had four types of blocks formed from the orthogonal combination of task and saliency, each block containing one condition (see Fig. 1). There were two target-salient blocks: (1) identify the global letters in stimuli where global information is more salient than local information (Fig. 1, bottom pair) and (2) identify the local letters in stimuli where local information is more salient than global (Fig. 1, top pair). There were two distractor-salient blocks: (1) identify the global letters in a stimulus where the local letters were more salient (Fig. 1, top pair) and (2) identify the local letters in stimuli where the global letters were more salient (Fig. 1, bottom pair). The target level was blocked to allow participants to adopt a topdown set to the designated target level. ${ }^{1}$ The first two blocks and the last two blocks of the experiment were both either globally salient displays or locally salient displays. Each block had 48 trials. On half of the trials, the same letters

\footnotetext{
${ }^{1}$ Example scenario of block order: first block, target-salient condition, global task using the set of stimuli with global focus (Fig. 1, bottom pair); second block, distractor-salient condition, local task using the set of stimuli with global focus (Fig. 1, bottom pair); third block, distractorsalient condition, global task using the set of stimuli with local focus (Fig. 1, top pair); fourth block, target-salient condition, local task using the set of stimuli with local focus (Fig. 1, top pair).
} 
appeared on the global and local levels (congruent trials), whereas on the other half, there were different letters on the two levels (incongruent trials). Each pair of these blocks consisted of a global and a local identification task. The order of the blocks was randomized across participants. Each experimental trial started with a white fixation point presented for $2,000 \mathrm{~ms}$, followed by a 150 -ms presentation of a compound letter on a black background. The trial ended after the participant identified the letter $(\mathrm{H}$ or $\mathrm{S})$ on the target level (global or local) and gave a speeded response by pressing one of the two mouse buttons (one for each letter). The interstimulus interval was variable $(1-4 \mathrm{~s}$ from the response of the participant on one trial to the onset of the stimulus on the next trial) to avoid possible predictions of stimulus onset. The viewing distance was controlled with a chinrest at $65 \mathrm{~cm}$ from the monitor. Psychophysics Toolbox for MATLAB (Brainard, 1997; Kleiner, Brainard, \& Pelli, 2007; Pelli, 1997) was used for the presentation of the paradigm and the collection of the responses.

Response times (RTs) and performance accuracy were recorded. Incorrect responses were excluded from the analysis. RTs were screened for outliers after mean and standard deviation were estimated on the basis of a convolved exGaussian function fit to each participant's data. RTs over 3.5 standard deviations away from the mean were rejected (Heathcote, Popiel, \& Mewhort, 1991).

One difficulty for comparisons between younger and older participants is that older participants can show general deficits in processing, with generalized slowing potentially playing a major role in cognitive decline (Lindenberger \& Baltes, 1994; Salthouse, 2000). These generalized effects may differentially affect performance as the task conditions become harder, a result that can masquerade as a selective effect of the conditions on older, relative to younger, adults. In order to examine whether there is indeed a selective effect in the two age groups, we analyzed the efficiency data using $Z$-transformations. The aim of $Z$-transformations is to dissociate group differences from the effects of generalized slowing/decreases in processing efficiency by examining effects of task conditions normalized by the average performance for each participant (Faust, Balota, Spieler, \& Ferraro, 1999). Specifically, the difference of mean efficiency of all trials in one condition cell and the mean efficiency across all condition cells was normalized to the standard deviation of efficiency across all conditions cells within a participant. This procedure was repeated for each condition cell and participant separately

\section{Results}

Exploratory analysis indicated that there were lower accuracy rates for older than for young adults, which were driven mainly by errors in distractor-salient conditions (Fig. 2, right axis). In order to account for speed/accuracy trade-offs in the analysis, the data were also analyzed by combining RTs and accuracy into a single efficiency measure (RT/proportion correct; see Townsend \& Ashby, 1983), as well as analyzing $Z$-transformations (see the Method section for a detailed description of the approach for transforming data). ${ }^{2}$

\section{Summary outcome}

The central focus of the study was to examine whether older adults, relative to young adults, were more affected by salient incongruent distractors. Thus, it was of highest relevance to investigate the difference in performance between trials with salient incongruent distractors and those with salient congruent distractors - that is, congruency cost in the distractorsalient condition. Therefore, we summarize at first the outcome from efficiency and $Z$-transformed data pooled across visual field (VF) and task, before reporting main effects and interactions (Tables 1 and 2) to justify pooling the data.

A significant three-way interaction between saliency, congruency, and age (Table 2) suggests that there may be a differential effect of congruency for older adults. Performance on trials with low-salient targets (e.g., select the local element in a stimulus with a high-salient global distractor), in particular, may be difficult for older adults if they have reduced ability to suppress highsalient distractors - a result mimicking the effects of TMS on the left PPC (Mevorach et al., 2010). To quantify this, the effect cost of congruency was calculated for each individual for each target saliency condition from the difference in the $Z$-scores between incongruent and congruent trials (incongruent - congruent; see Fig. 3b). A two-way ANOVA was conducted with a withinsubjects factor of salience (target salient vs. distractor salient) and a between-subjects factor of age. This revealed significant main effects of salience and aging, $F(1,41)=38.82, p<.001$ and $F(1,41)=8.72, p=.005$, and a significant interaction, $F(1,41)=4.45, p=.041$ (Fig. 3b). Further $t$-tests revealed that there was a reliable effect of age group on performance in the distractor-salient condition, but not in the target-salient condition, $t(1,41)=-2.11, p=.041$, and $t(1,41)=0.51, p=.510$, for distractor-salient and target-salient conditions, respectively. Thus, the older participants had a larger congruency effect, as compared with the young group, but only when low-salient targets had to be selected and high-salient distractors ignored (e.g., select the local element in a stimulus weighted to the global level). Importantly, as we present in the main analysis (see below), the increased congruency effect was not specific

\footnotetext{
${ }^{2}$ To the best of our knowledge, this is the first time that analyses based on $Z$-transformation have been conducted on measures of performance efficiency rather than RT. However, since efficiency is likely to have the same distribution as the RT data from which it is derived, it should be applicable here. In addition, to have analyzed the RT data alone would have been to miss the critical trade-off in accuracy in older participants.
} 


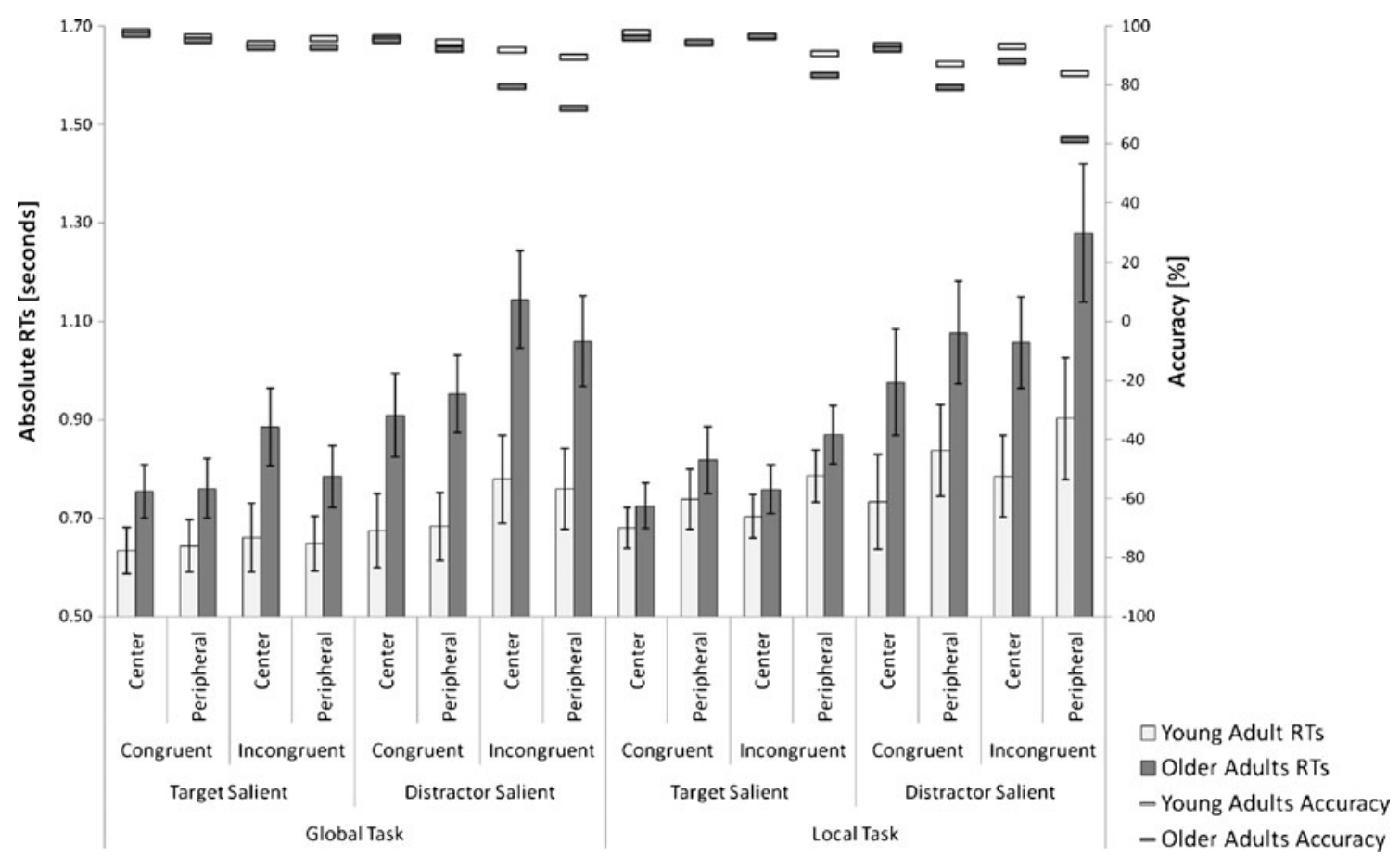

Fig. 2 Mean response times (RTs; $\pm 95 \%$ confidence interval) and accuracy as a function of congruency, saliency, and level of identification for younger and older adults

to a particular level of processing (local or global) and, therefore, indicates a general problem in suppressing high-salient distractors irrespective of the level of form involved.

Separate from the three-way interaction between saliency, congruency, and age, there was an overall effect of task and a reliable interaction between task and age (Table 2). The main effect of task occurred because, overall, responses to global targets were more efficient than responses to local targets. However, this effect varied with age (Fig. 4a). Relative to the overall average of performance for their age group, the young participants showed a larger difference between the global and local tasks (relatively fast for global and relatively slow for local, a $Z$ difference of 0.67 in efficiency), when compared with the older participants (a $Z$ difference of 0.27 in efficiency), $F(1,41)=5.35, p=.026$. Interestingly, the contrasting variation in performance across the age groups as a function of the task (Fig. 4a) went in the opposite direction to their respective variation as a function of stimulus saliency (Fig. 4b). We take up this point in the Discussion section below.

\section{Main analysis}

The experiment also replicated the expected main effects. For better transparency of the data, we now present main effects with supporting interactions for absolute RTs, efficiency, and $z$-transformed data. Finally, we present evidence for justifying the pooling of the data between left and right VFs into one common measure, peripheral vision.

\section{Absolute RT}

A five-way ANOVA was carried out on the absolute RTs and accuracy, with the within-subjects factors being target task (select global or local targets), saliency (target salient [e.g., global task with global saliency] vs. distractor salient [e.g., global task with local saliency]), congruency (congruent vs. incongruent), and VF (central vs. peripheral) (Fig. 2). There was a between-subjects factor of age group (young adults vs. older adults). $F$ - and $p$-values for significant main effects and interactions are displayed in Table 1.

Overall, RTs were shorter and accuracy higher in the global task than in the local task (796 vs. $858 \mathrm{~ms}$ ), on target-salient, as compared with distractor-salient, trials ( 740 vs. $913 \mathrm{~ms}$ ), on congruent, as compared with incongruent, trials (787 vs. $866 \mathrm{~ms}$ ), for central versus peripheral presentations (804 vs. $850 \mathrm{~ms}$ ), and in young, as compared with older, adults (728 vs. $926 \mathrm{~ms})$.

The highest order interaction in the RT data was between task, congruency, VF, and age group (Table 1). We estimated the cost of congruency relative to congruent trials from the difference in RTs for each task and VF condition separately within each group. We then analyzed a three-way ANOVA with the within-subjects factors of task and VF and the between-subjects-factor of age (Fig. 5). There were reliable main effects of age, $F(1,41)=8.18, p=.007$, task, $F(1,41)=$ $3.96, p=.053$, and VF, $F(1,41)=3.44, p=.071$. Significant interactions were observed between task and VF, $F(1,41)=$ 
Table $1 F$ - and $p$-values for significant main effects and interactions from a five-way ANOVA over the mean response times (RTs) and accuracy

\begin{tabular}{|c|c|c|c|c|c|c|c|}
\hline \multirow[t]{2}{*}{ Interaction } & & \multicolumn{3}{|l|}{ RTs } & \multicolumn{3}{|c|}{ Accuracy } \\
\hline & & $F$-Value & $p$-Value & Partial $\eta^{2}$ & $F$-Value & $p$-Value & Partial $\eta^{2}$ \\
\hline \multirow[t]{5}{*}{ Main Effects } & Task & 15.86 & $<.001$ & .279 & 9.83 & .003 & .193 \\
\hline & Saliency (Sal) & 71.88 & $<.001$ & .637 & 61.63 & $<.001$ & .600 \\
\hline & Congruency (Cong) & 99.96 & $<.001$ & .709 & 47.87 & $<.001$ & .539 \\
\hline & Visual field (VF) & 47.66 & $<.001$ & .538 & 81.43 & $<.001$ & .665 \\
\hline & Age & 20.93 & $<.001$ & & 7.73 & .008 & \\
\hline \multirow[t]{8}{*}{ General Interactions } & Task $\times$ Sal & 7.15 & .011 & .149 & 4.66 & .037 & .102 \\
\hline & Sal $\times$ Cong & 35.58 & $<.001$ & .465 & 11.64 & .001 & .221 \\
\hline & Task $\times$ VF & 78.51 & $<.001$ & .657 & 35.90 & $<.001$ & .467 \\
\hline & $\mathrm{Sal} \times \mathrm{VF}$ & 4.66 & .037 & .102 & 20.87 & $<.001$ & .337 \\
\hline & Cong $\times$ VF & - & & & 10.56 & .002 & .205 \\
\hline & Task $\times \mathrm{Sal} \times \mathrm{VF}$ & - & & & 5.01 & .031 & .109 \\
\hline & Task $\times$ Sal $\times$ Cong & - & & & 5.86 & .020 & .125 \\
\hline & Task $\times$ Cong $\times$ VF & 17.90 & $<.001$ & .304 & 8.76 & .005 & .176 \\
\hline \multirow[t]{10}{*}{ Interactions with Age } & Task $\times$ Age & - & & & - & & \\
\hline & Sal $\times$ Age & 19.55 & $<.001$ & .323 & 12.08 & .001 & .228 \\
\hline & Cong $\times$ Age & 13.33 & .001 & .245 & 14.11 & .001 & .256 \\
\hline & $\mathrm{VF} \times$ Age & - & & & 13.71 & .001 & 251 \\
\hline & Task $\times$ Sal $\times$ Age & 4.40 & .042 & .097 & - & & \\
\hline & Sal $\times$ Con $\times$ Age & 3.75 & .060 & .084 & 8.64 & .005 & .174 \\
\hline & Task $\times \mathrm{VF} \times$ Age & 6.06 & .018 & .129 & - & & \\
\hline & Sal $\times \mathrm{VF} \times$ Age & - & & & 5.68 & .022 & .122 \\
\hline & Cong $\times$ VF $\times$ Age & - & & & 7.33 & .010 & .152 \\
\hline & Task $\times$ Cong $\times$ VF $\times$ Age & 6.85 & .012 & .143 & - & & \\
\hline
\end{tabular}

The within-subjects factors included task (select global/local targets), saliency level (target-salient [e.g., global task with global saliency] vs. distractor-salient [e.g., global task with local saliency]), congruency (congruent vs. incongruent) and visual field (central vs. peripheral). The between-subjects factor was age group (young adults vs. older adults)

$20.71, p<.001$, and task, VF, and age, $F(1,41)=5.60, p=.023$. The three-way interaction was further broken down for each VF, using two separate two-way ANOVAs with the within-subjects factor of task and a between-subjects factor of age. The analysis for data with peripheral stimuli showed no significant effects [age, $F(1,41)=3.08, p=.087$; task, $F(1,41)=3.39, p=.073$; task $\times$ age, $F(1,41)=1.14, p=.291]$, whereas the analysis for data for centrally presented stimuli generated a significant main effect of task, $F(1,41)=24.82, p<.001$, and an interaction between task and age, $F(1,41)=6.06, p=.018$. A post hoc analysis revealed that the interaction was mainly driven by larger congruency effects in the global task with central presentation for older adults, relative to young adults $[F(1,41)=12.16, p=.001$, and $F(1,41)=0.525, p=.473$, congruency cost at global and local levels for central presentation, respectively].

\section{Efficiency}

Apart from the high-order interaction (task $\times$ congruency $\times \mathrm{VF} \times$ age), there was an additional three-way interaction (saliency $\times$ congruency $\times$ age) that approached significance in the RT data,
$F(1,41)=3.75, p=.060$, and this was also highly significant in the accuracy data, $F(1,41)=8.64, p=.005$ (Table 1$)$. In order to provide an overall analysis accounting for any speed/accuracy trade-offs in the analysis, the data were analyzed by combining RTs and accuracy into a single efficiency measure (RT/proportion correct; see Townsend \& Ashby, 1983).

Performance was assessed in mixed design ANOVAs using the mean efficiency data for each participant. The withinsubjects factors were task (select global or local targets), saliency (target salient [e.g., global task with global saliency] vs. distractor salient [e.g., global task with local saliency]), congruency (congruent vs. incongruent), and VF (central vs. peripheral). The between-subjects factor was age group (young adults vs. older adults). $F$ - and $p$-values for significant main effects and interactions are displayed in Table 2.

There was a four-way interaction between saliency, congruency, VF, and age, which suggests again that there may be a differential effect of congruency for older adults. To quantify this, the effect of congruency was calculated from the difference in mean efficiency for the congruent and incongruent conditions for each variation in target saliency and VF, for each age 
Table 2 Significance levels ( $F$ - and $p$-values) for the main effects and interactions involving the factors of task, saliency (Sal), congruency (Cong), visual field (VF), and age

\begin{tabular}{|c|c|c|c|c|c|c|c|}
\hline \multirow[t]{2}{*}{ Interaction } & & \multicolumn{3}{|c|}{ Efficiency (RT/Accuracy) } & \multicolumn{3}{|l|}{$Z$-score } \\
\hline & & $F$-Value & $p$-Value & Partial $\eta^{2}$ & $F$-Value & $p$-Value & Partial $\eta^{2}$ \\
\hline \multirow[t]{5}{*}{ Main Effects } & Task & 20.82 & $<.001$ & .337 & 29.81 & $<.001$ & .421 \\
\hline & Sal & 54.76 & $<.001$ & .572 & 188.16 & $<.001$ & .821 \\
\hline & Cong & 28.71 & $<.001$ & .412 & 166.09 & $<.001$ & .802 \\
\hline & $\mathrm{VF}$ & 43.10 & $<.001$ & .512 & 147.17 & $<.001$ & .782 \\
\hline & Age & 22.76 & $<.001$ & & - & & \\
\hline \multirow[t]{9}{*}{ General Interactions } & Task $\times$ Sal & 17.93 & $<.001$ & .304 & - & & \\
\hline & Sal $\times$ Cong & 13.82 & $<.001$ & .252 & 38.82 & $<.001$ & .486 \\
\hline & Task $\times$ VF & 32.83 & $<.001$ & .445 & 140.98 & $<.001$ & .775 \\
\hline & $\mathrm{Sal} \times \mathrm{VF}$ & 15.81 & $<.001$ & .278 & 43.58 & $<.001$ & .515 \\
\hline & Task $\times$ Sal $\times$ VF & 9.83 & .003 & .193 & 18.69 & $<.001$ & .313 \\
\hline & Cong $\times$ VF & 9.17 & .004 & .183 & - & & \\
\hline & Task $\times$ Cong $\times$ VF & 12.45 & .001 & .233 & 13.06 & .001 & .242 \\
\hline & Sal $\times$ Cong $\times$ VF & 7.83 & .008 & .160 & - & & \\
\hline & Task $\times$ Sal $\times$ Cong $\times$ VF & 4.36 & .043 & .096 & - & & \\
\hline \multirow[t]{12}{*}{ Interactions with Age } & Task $\times$ Age & - & & & 5.35 & .026 & .115 \\
\hline & Sal $\times$ Age & 21.42 & $<.001$ & .343 & 8.72 & .005 & .175 \\
\hline & Cong $\times$ Age & 11.53 & .002 & .220 & - & & \\
\hline & $\mathrm{VF} \times$ Age & 11.12 & .002 & .213 & - & & \\
\hline & Task $\times$ Sal $\times$ Age & 8.27 & .006 & .168 & - & & \\
\hline & Sal $\times$ Con $\times$ Age & 7.68 & .008 & .158 & 4.45 & .041 & .098 \\
\hline & Task $\times$ VF $\times$ Age & 9.99 & .003 & .196 & - & & \\
\hline & $\mathrm{Sal} \times \mathrm{VF} \times$ Age & 7.86 & .008 & .161 & - & & \\
\hline & Task $\times$ Sal $\times$ VF $\times$ Age & 5.54 & .023 & .119 & - & & \\
\hline & Congruency $\times \mathrm{VF} \times$ Age & 6.14 & .017 & .130 & - & & \\
\hline & Task $\times$ Cong $\times \mathrm{VF} \times$ Age & 7.13 & .011 & .148 & & & \\
\hline & Sal $\times$ Cong $\times$ VF $\times$ Age & 5.27 & .027 & .114 & - & & \\
\hline
\end{tabular}

a

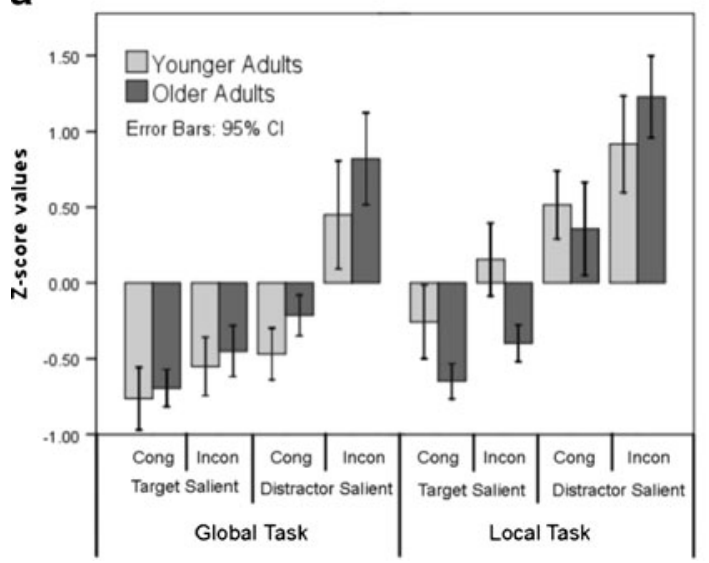

b

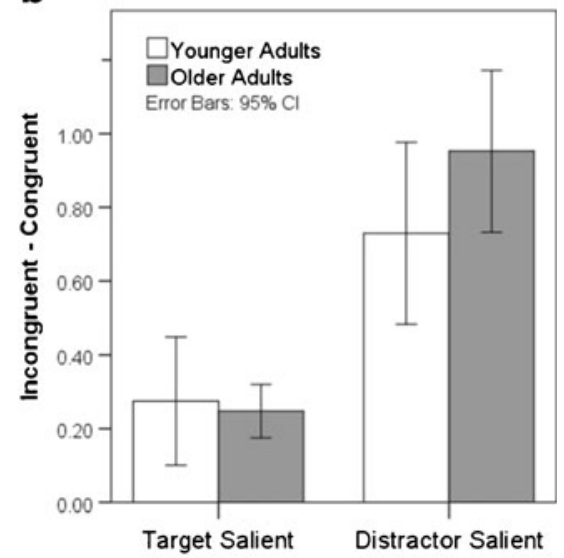

Fig. 3 a Mean $Z$-score values ( $\pm 95 \%$ confidence interval) as a function of congruency, saliency, and level of identification for young and older adults. Values indicate the difficulty ( -1 being easiest, +1 being most difficult) of a condition in relation to the averaged efficiency across all conditions (baseline). b Congruency cost calculated from the $Z$-score difference between incongruent and congruent trials for target-salient and distractor-salient conditions separately for young and older adults 


\section{a}

b

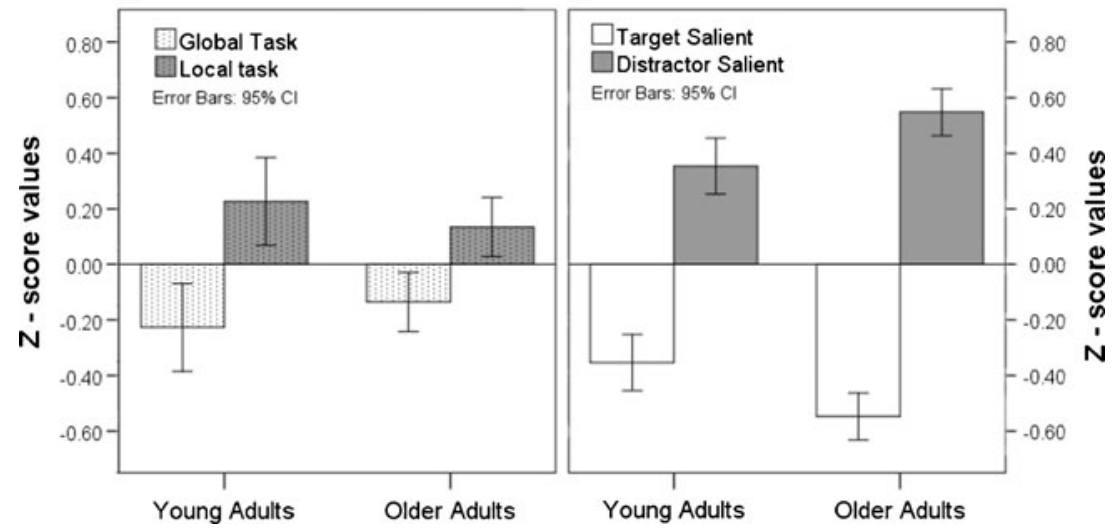

Fig. 4 Mean $Z$-score values ( $\pm 95 \%$ confidence interval) a as a function of task (global/local) and $\mathbf{b}$ as a function of target saliency (target-salient/ distractor-Salient) for young and older adults

group (see Fig. 6). A three-way ANOVA was estimated with the within-subjects factors of salience (target-salient vs. distractorsalient) and VF (central vs. peripheral presentation) and the between-subjects factor of age group. All main effects and interactions were significant. Further breakdown of the higher order interaction (saliency $\times \mathrm{VF} \times$ age) included two separate two-way ANOVAs, one for each VF (central and peripheral presentation), with the within-subjects factor of saliency (target salient vs. distractor salient) and the between-subjects factor of age. Stimuli presented centrally produced main effects of saliency, $F(1,41)=8.37, p=.006$, and age, $F(1,41)=$ $8.43, p=.006$, and a marginally significant interaction between saliency and age, $F(1,41)=3.94, p=.054$. There was a similar set of results with peripheral presentations [main effects of age, $F(1,41)=10.78, p=.002$, and saliency, $F(1,41)=$ $13.20, p=.001$, and an age $\times$ saliency interaction, $F(1,41)=$ $7.80, p=.008]$. For both central and peripheral stimuli, older

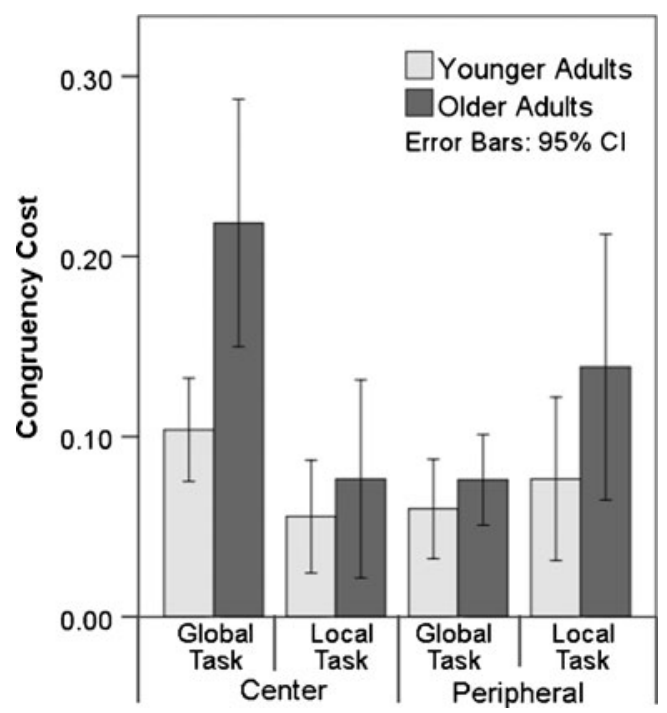

Fig. 5 Mean congruency cost $( \pm 95 \%$ confidence interval) as a function of task and visual field for young and older adults separately participants showed stronger effects of congruency than did younger participants, particularly when the target was low salient and the distractor high salient.

\section{Z-transformations}

Performance was assessed in mixed design ANOVAs over the mean Z-transformation data, with the within-subjects factors of task (select global or local targets), saliency (target salient [e.g., global task with global saliency] vs. distractor salient [e.g., global task with local saliency]), congruency (congruent vs. incongruent), and VF (central vs. peripheral). The between-subjects factor was age group (young adults vs. older adults). $F$ - and $p$-values for significant main effects and interactions are displayed in Table 2.

In these analyses, all higher-order interactions, including VF, became nonsignificant, suggesting that interactions including

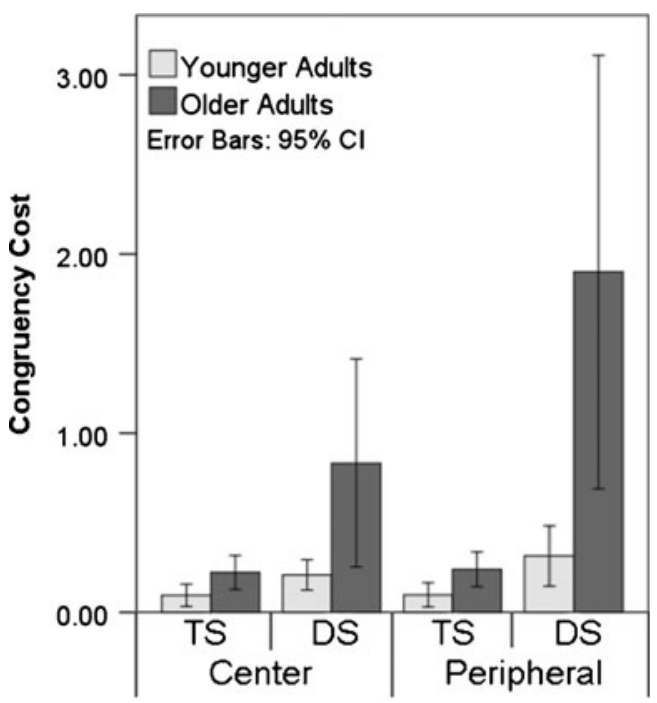

Fig. 6 Mean congruency cost ( $\pm 95 \%$ confidence interval) as a function of saliency (target Salient [TS] vs. distractor salient [DS]) and visual field (center vs. peripheral) for young and older adults separately 
$\mathrm{VF}$ in the nontransformed efficiency data were probably influenced by generalized effects of aging. The three-way interaction between saliency, congruence, and age (see above and Table 2) remained significant after adjustments for effects of generalized slowing, suggesting that the differential effects of saliency processing between age groups were task specific.

\section{Left versus right visual field}

An ANOVA on RT data from the left and right field locations (excluding centrally presented stimuli) assessed whether there were differential effects of VF on performance. There was a reliable main effect of field, with RT values (as well as efficiency and $Z$-transformed data) lower to targets in the left field (left, $842 \mathrm{~ms}$; right, $858 \mathrm{~ms}$ ), $F(1,41)=5.39, p=.025$. However, there were no other significant interactions with other factors, including age group, and the data were subsequently pooled across hemispheric field (Fig. 2).

\section{Discussion}

The main finding was that, relative to young adults, older adults were more affected by salient incongruent distractors (producing higher congruency costs in the distractor-salient condition), and this held even with the analysis scaled for the effects of aging on overall efficiency (using $Z$-transformations). The effect sizes show that this was a relatively small effect, possibly because general slowing contributed to the age-related changes; nonetheless, it is relevant and significant. This age-related decline in the ability to select a lowsalient target in the presence of a high-salient distractor held for both levels of target identification, with both local and global stimuli (respectively, when the global or the local saliency of the distractor was high). Importantly, this increased congruency effect in distractor-salient displays cannot be attributed to generally heightened susceptibility to salience in old age. If heightened sensitivity to salience was driving the effect, our old participants should have also shown a difference in performance in the target-salient conditions (e.g., a reduced congruency effect when salient targets were reported, since older adults would be less sensitive to target saliency). We therefore conclude that performance in the old group most likely represents reduced down-regulated inhibition of saliency, encountered particularly under conditions where distractors are salient. We note that this result mimics the effects of TMS suppression reported by Mevorach et al. (2010), where the loss of inhibitory control was most evident with salient distractors.

Our findings are concordant with the inhibition deficit theory (Lustig et al., 2007), which posits that older adults are generally less able to inhibit unwanted information-although here we show, for the first time, that this applies to nonspatial selection of local and global forms. According to the inhibition deficit framework, early bottom-up responses to salient, exogenous stimuli require inhibitory mechanisms to limit processing when the stimuli are irrelevant (i.e., to ignore the conversation of nearby passengers while reading a newspaper in a train). Deficits in the efficiency of inhibiting irrelevant distractors may disrupt the ability to focus attention on stimuli of interest, resulting in the dilution of selection across distractors as well as targets. As was noted in the Introduction, deficits in filtering out distractors have been observed across a range of conditions, with different types of stimuli-for example, in reading (Carlson, Hasher, Zacks, \& Connelly, 1995), language comprehension and production (Burke, 1997; Burke \& Mackay, 1997; Tun, O'Kane, \& Wingfield, 2002), visual memory (Gazzaley, Cooney, Rissman, \& D'Esposito, 2005), spatial visual selection (Schlaghecken, Birak, \& Maylor, 2012; Watson \& Maylor, 2002), and nonspatial visual selection in the Stroop task (Davidson, Zacks, \& Williams, 2003; West \& Alain, 2000; West \& Bell, 1997). To our knowledge, this is the first study showing age-related deficits in inhibition in nonspatial selection of hierarchical forms. ${ }^{3}$

In addition, our findings link the inhibitory deficit theory with observations from neuroimaging. There is a striking parallel between our data and prior studies in which TMS was applied to the left PPC, to reduce its influence on suppressing perceptual representations of distractors (Mevorach et al., 2010; Mevorach et al., 2006b). Mevorach et al. reported that, across both local and global levels, low-salient targets became difficult to select after the left PPC received TMS, and this was associated with increased activation in the early occipital cortex. These data are consistent with low-salient targets being selected through modulated inhibition of highsalient distractors via the left PPC and with this top-down selection process being compromised with age. The data also fit with the posterior-anterior shift with aging model, which posits an age decline in the occipito-parietal networks involved in attention (Cabeza et al., 2004; Davis, Dennis, Daselaar, Fleck, \& Cabeza, 2008). We may speculate that the age-related decline in the suppression of salient information may be due to age-related decreases in the effectiveness of connectivity between occipital and parietal cortices. Irrespective of this, our behavioral data suggest that altered control of attention to low-salient signals may be a critical factor in cognitive aging and, at least in our results, something that is more important than alterations in the selection of local and global targets.

Problems in selecting low-salient stimuli may have been critical to findings from studies using distraction as a measure

\footnotetext{
${ }^{3}$ Note that the selection of a global stimulus, and ignoring of a local stimulus, cannot be explained in terms of spatial selection, since any "fitting" of a spatial window of attention on a global stimulus would also lead to local stimuli being selected.
} 
of top-down attentional control in aging. The inhibition of task-irrelevant information in aging has been assessed from responses to task-irrelevant abrupt onsets (Kramer, Hahn, Irwin, \& Theeuwes, 1999) and the inhibition of cued information in top-down visual search (Madden, Spaniol, Bucur, \& Whiting, 2007; Madden, Whiting, Cabeza, \& Huettel, 2004). Although there are results suggesting that there is preservation of top-down attentional control with aging (Kramer et al., 1999; Madden et al., 2004; Whiting, Madden, \& Babcock, 2007; Whiting, Madden, Pierce, \& Allen, 2005), this has not been established in cases where distractors have relatively high saliency (as compared with targets) (Kramer, Hahn, Irwin, \& Theeuwes, 2000; Madden et al., 2004). The present results go beyond these data by suggesting that there are impairments in rejecting high-salient distractors at different levels of stimulus representation. It is perfectly possible that other forms of top-down processing, such as the guidance of attention from positive expectancies of targets, remain intact.

One somewhat different account of the present results can also be put forward. This is that older adults suffered more interference from salient distractors because they had more efficient parallel processing of both levels of the hierarchical forms. This more efficient parallel processing would mean that distractors are processed more deeply and, thus, create more interference. However, on this account, we would expect that RTs on congruent trials would be notably short for older adults, since they would gain more from redundancy at the distractor level. There was no evidence for this. The failure to find an increased benefit on congruent trials for older participants also goes against the idea that older adults show increased congruency effects due to congruent trials being speeded.

Aside from the effects of saliency, the old and young age groups differed in how their performance varied in the global and local identification tasks. The young participants showed relatively large differences in performance in the global, as compared with the local, task, when compared with their overall performance. The older participants showed relatively small changes between the global and local tasks, as compared with their overall performance. On the other hand, the older participants showed larger variation than the young participants as stimulus saliency changed (Fig. 4a, b). These data suggest that, for older but not for young participants, effects of saliency produce stronger shifts in performance than do effects of task (global vs. local). Our results, stressing the effects of saliency across different levels of form, also help to explain previous inconsistencies in the literature, where opposite effects of aging have been reported under conditions where the saliency of the local and global forms was varied (cf. MullerOehring et al., 2007; Roux \& Ceccaldi, 2001). Our data also cannot be linked to an argument that younger participants showed stronger effects of global precedence than did older participants (Roux \& Ceccaldi, 2001). Note that our effects occurred across the local and global recognition tasks, and we would then have expected a relatively larger congruency effect in young participants in the local task with distractor-salient stimuli. We observed the opposite (Fig. 3a).

One limitation of the present study is that, in the present procedure, it is difficult to separate problems in selecting the appropriate perceptual level of the target from difficulties encountered in selecting the response to the target when the distractor had high saliency; and indeed, both poor perceptual and response selection may contribute to the age-related effects we report. Note, however, that the neuroimaging data reported by Mevorach et al. (2010) strongly point to perceptual selection being challenged when the target has low saliency and the distractor high perceptual saliency (with changes found in early occipital brain regions). Future work needs to try and tease apart the age effects on perceptual and response selection, perhaps building on the imaging work, which provides a clear framework for evaluating the neuroanatomical basis of selection by saliency.

Finally, the advantage of left over right VF was unsurprising. Prior studies have reported a left-VF advantage (Orr \& Nicholls, 2005), most probably reflecting right-hemisphere dominance for attentional processing (Siman-Tov et al., 2007). Critically, this did not interact with age. Furthermore, there were no interactions between VFs (center vs. peripheral) and age, which provided evidence that the results cannot be explained with loss of visual acuity in the periphery. We conclude that age has a selective effect on rejecting highsalient distraction, an ability associated with distractor suppression through the left PPC in our task.

Author note This work was carried out in partial completion of a Ph.D. at the University of Birmingham by Kamen Tsvetanov. The work was supported by grants from the BBSRC and ESRC (U.K.).

\section{References}

Allen, H. A., Humphreys, G. W., \& Matthews, P. M. (2008). A neural marker of content-specific active ignoring. Journal of Experimental Psychology. Human Perception and Performance, 34, 286297.

Allen, H. A., \& Payne, H. (2011). Similar behaviour, different brain patterns: Age-related changes in neural signatures of ignoring. Neuroimage.

Brainard, D. H. (1997). The psychophysics toolbox. Spatial Vision, 10, 433-436.

Braithwaite, J. J., Humphreys, G. W., \& Hulleman, J. (2005). Color-based grouping and inhibition in visual search: Evidence from a probe detection analysis of preview search. Perception \& Psychophysics, 67(1), 81-101. doi:10.3758/BF03195014

Bruyer, R., Scailquin, J. C., \& Samson, D. (2003). Aging and the locus of the global precedence effect: A short review and new empirical data. Experimental Aging Research, 29, 237-268.

Burke, D. M. (1997). Language, aging, and inhibitory deficits: Evaluation of a theory. The Journals of Gerontology. Series B, Psychological Sciences and Social Sciences, 52, 254-264. 
Burke, D. M., \& Mackay, D. G. (1997). Memory, language, and ageing. Philosophical Transactions of the Royal Society of London. Series B, Biological Sciences, 352, 1845-1856.

Cabeza, R., Daselaar, S. M., Dolcos, F., Prince, S. E., Budde, M., \& Nyberg, L. (2004). Task-independent and task-specific age effects on brain activity during working memory, visual attention and episodic retrieval. Cerebral Cortex, 14, 364-375.

Carlson, M. C., Hasher, L., Zacks, R. T., \& Connelly, S. L. (1995). Aging, distraction, and the benefits of predictable location. Psychology and Aging, 10, 427-436.

Corbetta, M., \& Shulman, G. L. (2002). Control of goal-directed and stimulus-driven attention in the brain. Nature Reviews Neuroscience, 3, 201-215.

Davidson, D. J., Zacks, R. T., \& Williams, C. C. (2003). Stroop interference, practice, and aging. Neuropsychology, Development, and Cognition. Section B, Aging, Neuropsychology and Cognition, 10, 85-98.

Davis, S. W., Dennis, N. A., Daselaar, S. M., Fleck, M. S., \& Cabeza, R. (2008). Que PASA? The posterior-anterior shift in aging. Cerebral Cortex, 18, 1201-1209.

Dent, K., Allen, H. A., Braithwaite, J. J., \& Humphreys, G. W. (2012). Parallel distractor rejection as a binding mechanism in search. Frontiers in Psychology, 3, 278. doi:10.3389/fpsyg.2012.00278

Faust, M. E., Balota, D. A., Spieler, D. H., \& Ferraro, F. R. (1999). Individual differences in information-processing rate and amount: Implications for group differences in response latency. Psychological Bulletin, 125, 777-799.

Gazzaley, A., Cooney, J. W., Rissman, J., \& D'Esposito, M. (2005). Top-down suppression deficit underlies working memory impairment in normal aging. Nature Neuroscience, 8, 1298-1300.

Hasher, L., Stoltzfus, E. R., Zacks, R. T., \& Rypma, B. (1991). Age and inhibition. Journal of Experimental Psychology: Learning, Memory, and Cognition, 17, 163-169.

Heathcote, A., Popiel, S., \& Mewhort, D. J. (1991). Analysis of response time distributions: An example using the Stroop task. Psychological Bulletin, 109, 340-347.

Hodsoll, J., Mevorach, C., \& Humphreys, G. W. (2009). Driven to less distraction: rTMS of the right parietal cortex reduces attentional capture in visual search. Cerebral Cortex, 19, 106-114.

Humphreys, G. W., Stalmann, B. J., \& Olivers, C. (2004). An analysis of the time course of attention in preview search. Perception \& Psychophysics, 66, 713-730.

Kleiner, M., Brainard, D., \& Pelli, D. (2007). What's new in psychtoolbox3? perception 36. Ref Type: Abstract.

Kramer, A. F., Hahn, S., Irwin, D. E., \& Theeuwes, J. (1999). Attentional capture and aging: Implications for visual search performance and oculomotor control. Psychology and Aging, 14, 135-154.

Kramer, A. F., Hahn, S., Irwin, D. E., \& Theeuwes, J. (2000). Age differences in the control of looking behavior: Do you know where your eyes have been? Psychological Science, 11, 210-217.

Lindenberger, U., \& Baltes, P. B. (1994). Sensory functioning and intelligence in old age: A strong connection. Psychology and Aging, 9, 339-355.

Lustig, C., Hasher, L., \& Zacks, R. (2007). Inhibitory deficit theory: Recent developments in a "new view". In D. S. Gorfein \& C. M. MacLeod (Eds.), The place of inhibition in cognition (pp. 145162). Washington: American Psychological Association.

Lux, S., Marshall, J. C., Ritzl, A., Weiss, P. H., Pietrzyk, U., Shah, N. J., et al. (2004). A functional magnetic resonance imaging study of local/global processing with stimulus presentation in the peripheral visual hemifields. Neuroscience, 124, 113-120.

Madden, D. J. (2007). Aging and visual attention. Current Directions in Psychological Science, 16, 70-74.

Madden, D. J., Gottlob, L. R., \& Allen, P. A. (1999). Adult age differences in visual search accuracy: Attentional guidance and target detectability. Psychology and Aging, 14, 683-694.
Madden, D. J., Spaniol, J., Bucur, B., \& Whiting, W. L. (2007). Agerelated increase in top-down activation of visual features. Quarterly Journal of Experimental Psychology (Hove), 60, 644-651.

Madden, D. J., Whiting, W. L., Cabeza, R., \& Huettel, S. A. (2004). Age-related preservation of top-down attentional guidance during visual search. Psychology and Aging, 19, 304-309.

Mevorach, C., Hodsoll, J., Allen, H., Shalev, L., \& Humphreys, G. (2010). Ignoring the elephant in the room: A neural circuit to downregulate salience. Journal of Neuroscience, 30, 6072-6079.

Mevorach, C., Humphreys, G. W., \& Shalev, L. (2005). Attending to local form while ignoring global aspects depends on handedness: Evidence from TMS. Nature Neuroscience, 8, 276-277.

Mevorach, C., Humphreys, G. W., \& Shalev, L. (2006a). Effects of saliency, not global dominance, in patients with left parietal damage. Neuropsychologia, 44, 307-319.

Mevorach, C., Humphreys, G. W., \& Shalev, L. (2006b). Opposite biases in salience-based selection for the left and right posterior parietal cortex. Nature Neuroscience, 9, 740-742.

Mevorach, C., Humphreys, G. W., \& Shalev, L. (2009a). Reflexive and preparatory selection and suppression of salient information in the right and left posterior parietal cortex. Journal of Cognitive Neuroscience, 21, 1204-1214.

Mevorach, C., Shalev, L., Allen, H. A., \& Humphreys, G. W. (2009b). The left intraparietal sulcus modulates the selection of low salient stimuli. Journal of Cognitive Neuroscience, 21, 303-315.

Muller-Oehring, E. M., Schulte, T., Raassi, C., Pfefferbaum, A., \& Sullivan, E. V. (2007). Local-global interference is modulated by age, sex and anterior corpus callosum size. Brain Research, 1142, 189-205.

Olivers, C. N., \& Humphreys, G. W. (2003). Visual marking inhibits singleton capture. Cognitive Psychology, 47, 1-42.

Orr, C. A., \& Nicholls, M. E. (2005). The nature and contribution of space- and object-based attentional biases to free-viewing perceptual asymmetries. Experimental Brain Research, 162, 384-393.

Pelli, D. G. (1997). The VideoToolbox software for visual psychophysics: Transforming numbers into movies. Spatial Vision, 10, 437-442.

Plude, D. J. (1990). Aging, feature integration, and visual selective attention. Advances in Psychology, 69, 467-487.

Roux, F., \& Ceccaldi, M. (2001). Does aging affect the allocation of visual attention in global and local information processing? Brain and Cognition, 46, 383-396.

Salthouse, T. A. (2000). Aging and measures of processing speed. Biological Psychology, 54, 35-54.

Schlaghecken, F., Birak, K. S., \& Maylor, E. A. (2012). Age-related deficits in efficiency of low-level lateral inhibition. Frontiers in Human Neuroscience, 6, 102.

Siman-Tov, T., Mendelsohn, A., Schonberg, T., Avidan, G., Podlipsky, I., Pessoa, L., et al. (2007). Bihemispheric leftward bias in a visuospatial attention-related network. Journal of Neuroscience, 27. 11271-11278

Theeuwes, J. (1992). Perceptual selectivity for color and form. Perception \& Psychophysics, 51, 599-606.

Theeuwes, J. (2005). Irrelevant singletons capture attention. In L. Itti, G. Rees, \& J. K. Tsotsos (Eds.), Neurobiology of attention (pp. 418-424). San Diego: Elsevier.

Tipper, S. P., \& Cranston, M. (1985). Selective attention and priming: Inhibitory and facilitatory effects of ignored primes. Quarterly Journal of Experimental Psychology. A, Human Experimental Psychology, 37, 591-611.

Townsend, J. T., \& Ashby, F. G. (1983). The stochastic modelling of elementary psychological processes. Cambridge University Press.

Tun, P. A., O'Kane, G., \& Wingfield, A. (2002). Distraction by competing speech in young and older adult listeners. Psychology and Aging, 17, 453-467.

Watson, D. G., \& Humphreys, G. W. (1997). Visual marking: Prioritizing selection for new objects by top-down attentional inhibition of old objects. Psychological Review, 104, 90-122. 
Watson, D. G., \& Humphreys, G. W. (2000). Visual marking: Evidence for inhibition using a probe-dot detection paradigm. Perception \& Psychophysics, 62, 471-481.

Watson, D. G., \& Maylor, E. A. (2002). Aging and visual masking: Selective deficits for moving stimuli. Psychology and Aging, 17, 321-339.

West, R., \& Alain, C. (2000). Age-related decline in inhibitory control contributes to the increased Stroop effect observed in older adults. Psychophysiology, 37, 179-189.

West, R., \& Bell, M. A. (1997). Stroop color-word interference and electroencephalogram activation: Evidence for age-related decline of the anterior attention system. Neuropsychology, 11, 421-427.
Whiting, W. L., Madden, D. J., \& Babcock, K. J. (2007). Overriding age differences in attentional capture with top-down processing. Psychology and Aging, 22, 223-232.

Whiting, W. L., Madden, D. J., Pierce, T. W., \& Allen, P. A. (2005). Searching from the top-down: Ageing and attentional guidance during singleton detection. Quarterly Journal of Experimental Psychology. A, Human Experimental Psychology, 58, 72-97.

Wolfe, J. M., Butcher, S. J., Lee, C., \& Hyle, M. (2003). Changing your mind: On the contributions of top-down and bottom-up guidance in visual search for feature singletons. Journal of Experimental Psychology. Human Perception and Performance, 29, 483-502. 